\title{
Empoderamiento de personas con diversidad funcional intelectual en el escenario universitario: aportaciones desde la psicopedagogía
}

Enviado: 28 de julio de 2020 / Aceptado: 29 de septiembre de 2020

Publicado: 30 de diciembre de 2020

CRISTINA SUÁREZ RODRÍGUEZ

Universidad de Málaga, España suarez@uma.com

MARÍA JOSÉ ALCALÁ DEL OLMO FERNÁNDEZ

Departamento de Didáctica y Organización Escolar,

Universidad de Málaga, España

mjalcaladelolmo@uma.com

\section{RESUMEN}

La inclusión educativa representa una apuesta por atender a la diversidad, a partir de un tejido curricular accesible con el que responder a las necesidades del alumnado altamente diverso. En el escenario universitario, este reto implica el diseño de espacios de aprendizaje centrados en los/as estudiantes, como modo de contribuir a la superación de barreras que se oponen a la construcción de conocimientos.

En respuesta a determinadas leyes y políticas, los centros de Educación Superior han establecido servicios de apoyo para satisfacer las demandas de su alumnado, convirtiéndose en espacios destinados a garantizar una plena inclusión socio-laboral, recurriendo, para ello, a diversas estrategias, como las vinculadas a las manifestaciones artísticas y el mundo cultural en general.

\section{ABSTRACT}

Empowerment of people with intellectual functional diversity in the university stage: contributions from psychopedagogy

Educational inclusion represents a commitment to address diversity, based on an accessible curricular fabric with which to respond to the needs of highly diverse students. In the university scenario, this challenge involves the design of learning spaces focused on the students, as a way to contribute to the overcoming barriers that are opposed to the construction of knowledge.

In response to certain laws and policies, the Higher Education centers have established support services to meet the demands of their students, becoming spaces destined to guarantee full socio-labor inclu- 
Una de las disciplinas que mayor impacto ha recibido de las esencias de la educación inclusiva ha sido la psicopedagogía, lo que ha llevado a definirla como una parte consustancial de la misma, en un claro intento por identificar aquellas medidas educativas más eficaces para solventar problemas de aprendizaje y conceder una respuesta efectiva a la diversidad.

A lo largo de este trabajo, se presenta el diseño de una intervención psicopedagógica llevada a cabo con un grupo de estudiantes con diversidad funcional intelectual que forman parte del Título Propio de Técnico Auxiliar en Entornos Culturales de la Universidad de Málaga. En el mismo, se ha reivindicado la perpetuidad de la educación inclusiva en los niveles formativos postobligatorios, como puente hacia el empoderamiento y conformación de la identidad personal y laboral de estos colectivos.

Palabras Clave: educación inclusiva, educación superior, diversidad estudiantil, expresión artística, empoderamiento del alumnado. sion, resorting, for this, to various strategies, such as those linked to artistic manifestations and the cultural world in general.

One of the disciplines that has received the greatest impact from the essences of inclusive education has been psychopedagogy, which has led it to be defined as an essential part of it, in a clear attempt to identify the most effective educational measures to solve learning problems and to provide an effective response to diversity.

Throughout this work, it is present the design of a psychopedagogical intervention carried out with a group of students with intellectual functional diversity who are part of the Title of Technical Assistant in Cultural Environments of the University of Malaga. In it, the perpetuation of inclusive education at postcompulsory educational levels has been claimed, as a bridge towards the empowerment and shaping of the personal and work identity of these groups.

Keywords: educational inclusion, higher education, student diversity, art expression, student empowerment.

\section{INTRODUCCIÓN}

Durante las últimas décadas, se ha generado un cambio significativo en la forma de definir las esencias y objetivos de la psicopedagogía. De ser delimitada como un ámbito de estudio resultante de la confluencia entre la psicología y la pedagogía, ha pasado a valorarse como una disciplina científica y profesional con gran arraigo en el panorama internacional.

Su presencia en la trayectoria formativa universitaria también ha sufrido alteraciones, dado que la Licenciatura que llevaba su nombre desapareció, siendo necesario el esfuerzo del profesorado universitario, que, interesado en visibilizar su importancia en la Educación Superior, contribuyó a recuperar en forma de Máster Oficial esta titulación, marcada por la necesidad de ofrecer a los futuros profesionales de la educación una formación con la que tomar conciencia de la necesidad de proporcionar a cada educando el trato equitativo que merece. 
Ello hace que las labores a emprender por los profesionales de la psicopedagogía formen parte del proceso instructivo de los estudiantes que deciden matricularse en estos estudios de posgrado, figurando, entre ellas, las referidas a la evaluación psicopedagógica, el diagnóstico de necesidades educativas, el análisis de estrategias de enseñanza y aprendizaje, el diseño y aplicación de la intervención psicopedagógica, la atención a la diversidad y la orientación educativa, vocacional y profesional (Ventura, 2015).

A lo largo de la historia, esta disciplina ha sido valorada como el principal espacio desde el que atender a las dos últimas funciones comentadas. De hecho, no se puede olvidar que la psicopedagogía emerge de las principales necesidades detectadas en las aulas, buscando, en todos los casos, incrementar la calidad de la atención educativa, percibiéndose en ella una doctrina que recurre a los fundamentos de la psicología como forma de enriquecer las tareas educativas y dotarlas de la mayor excelencia posible (Messi, Rossi y Ventura, 2016).

La educación, por tanto, se nutre de las aportaciones de la psicología para conceder respuesta a los intereses e inquietudes de los discentes en general, y, especialmente, de aquellos que presentan dificultades. Es precisamente al amparo de este planteamiento, en el que puede situarse la relevancia de la psicopedagogía en el marco de la escuela inclusiva, como forma de valorar los procesos de aprendizaje contextualizados, para decidir cuáles pueden ser los apoyos y medidas educativas más coherentes en cada caso.

En la actualidad, la implementación de la psicopedagogía va unida a la necesidad de reflexionar acerca de aquellas actuaciones que pueden ser más eficaces para el alumnado altamente diverso (Baraldi, 2016), con objeto de asumir una perspectiva más amplia, inclusiva y comprometida con las diferencias, buscando trascender la categorización y descripción de aquello que nos define y de lo que nos limita, para reconocer el valor de la comprensión holística de la alteridad en clave de respeto y tolerancia.

En este sentido, se admite que la educación inclusiva constituye una parte consustancial de la psicopedagogía (Demchenko, Rokosovyk y Volodymyr, 2017; Lorente y Sales, 2017), lo que lleva a sus profesionales a asumir responsabilidades para trabajar a favor del empoderamiento y la resiliencia de las personas con diversidad, con la intención de perfilar un autoconcepto positivo que propicie una efectiva inclusión educativa y social, trabajando, además de los aspectos cognitivos, aquellos relacionados con lo socio-afectivo y emocional, como ingredientes esenciales en la consecución del entusiasmo y la ilusión por aprender y trabajar.

Uno de los escenarios en los que mayor impacto ha tenido el paradigma de la inclusión educativa a lo largo de las últimas décadas ha sido el universitario, lo que ha llevado a la 
proliferación de Acuerdos Internacionales y redacción de documentos legislativos en los que tratan de plantearse medidas con las que asegurar el principio de igualdad de oportunidades en la Educación Superior (Collins, Azmat y Rentschler, 2018; Fernández-Fernández, VélizBriones y Ruiz-Cedeño, 2016; García y García, 2017; Lawrie et al., 2017; Moriña, 2020).

No obstante, en la comunidad científica ha podido denotarse que el colectivo que presenta más dificultades en el acceso, permanencia y egreso a su paso por la universidad ha sido el formado por los estudiantes con diversidad funcional intelectual, que suelen ser víctimas de la discriminación con una mayor crudeza, lo que requiere contar con profesionales comprometidos en ofrecer una formación capaz de dotar de las herramientas pedagógicas necesarias con las que propiciar la construcción autónoma de los aprendizajes como forma de progresar en el camino hacia la inclusión laboral.

En este contexto, se sitúa el Título Propio de Técnico Auxiliar en Entornos Culturales, en el que se fundamenta este trabajo, como oferta formativa impartida en la Universidad de Málaga desde su creación a partir del curso académico 2017-2018 (Martínez-Silvente y Rodríguez-Infante, 2019), en la que el arte se funde con la igualdad de género para ofrecer al alumnado con diversidad funcional intelectual aquellos instrumentos indispensables con los que facilitar la asimilación de conocimientos en paralelo a su adaptación al mundo laboral.

Iniciativas como estas enfatizan la importancia del profesorado universitario como profesional que debe crear situaciones estimulantes de aprendizaje que lleven a los estudiantes a pensar e indagar, en un escenario de encuentro compartido en el que la diversidad es elemento enriquecedor y garante en la conformación de una estructura universitaria sostenible e inclusiva (Bortolini, Óscar y Almeida, 2018; Carballo, Cotán y Spínola, 2019; Melero, Moriña y Perera, 2019; Moriña, 2020).

Con el propósito de mostrar las claves más significativas de la intervención psicopedagógica en la atención a la diversidad en el contexto universitario, en este trabajo, se ofrece el diseño de una propuesta inclusiva destinada a empoderar a las personas con diversidad funcional intelectual, y, por ende, dotarlas de estrategias con las que acceder al mercado laboral con las mejores condiciones posibles. El hilo conductor a lo largo de la misma ha sido el arte, como forma de manifestar sentimientos y emociones, alcanzar un desarrollo integral, y, con ello, una positiva inclusión socio-laboral. 


\section{PSICOPEDAGOGÍA Y ATENCIÓN A LA DIVERSIDAD EN EL CONTEXTO UNIVERSITARIO: INTEGRACIÓN TRANSVERSAL DE LA AUTODETERMINACIÓN EN LAS INTERVENCIONES PSICOPEDAGÓGICAS}

En un intento por definir el término psicopedagogía, es interesante recurrir a las aportaciones que, al respecto, establece la Real Academia Española (2020), que la vislumbra como una rama perteneciente a la psicología encargada del estudio y análisis de fenómenos psicológicos para determinar los métodos pedagógicos y didácticos más apropiados a cada caso. A partir de esta valoración, la psicopedagogía se percibe como una disciplina que acentúa el valor de la identificación e intervención con personas que presentan algún trastorno o alguna dificultad en el plano psicológico.

A lo largo de este trabajo, se ha querido subrayar la necesidad de adoptar una perspectiva holística de la psicopedagogía, que albergue una amplia amalgama de componentes, principalmente aquellos referidos al estudio de los procesos de aprendizaje humano, en espacios formales y contextualizados, junto a las alteraciones que pueden surgir durante los mismos, además de aquellas variables que, relativas a aspectos socio-afectivos y emocionales, inciden en los procesos de aprendizaje y en la adquisición de conocimientos.

Por ello, la intervención psicopedagógica que sustenta este trabajo, no podría tener éxito de no incluirse en la misma un componente emocional, lo que lleva a admitir que el profesional de la psicopedagogía no debe ceñirse de forma exclusiva a la detección y clasificación de las alteraciones, sino que más bien debe esforzarse en la búsqueda de las respuestas educativas más coherentes en el marco de la atención a la diversidad, estableciendo nexos de unión con variables afectivas y emocionales (Cejudo, 2017), como factores enriquecedores de la praxis educativa principal.

La importancia de este tipo de aspectos aumenta en el caso de estudiantes de avanzada edad, con deseos de acceder al mercado laboral, y que, al mismo tiempo presentan alguna discapacidad, lo que les convierte en sectores más vulnerables para desplegar íntegramente sus potencialidades en los espacios profesionales.

A este respecto, no resulta baladí subrayar la importancia de la psicopedagogía como una forma de brindar apoyo, comprensión y acompañamiento durante la trayectoria formativa en la universidad a estudiantes con dificultades, en un intento por contribuir a la conformación de un autoconcepto equilibrado y una positiva inclusión socio-laboral. 
La intervención psicopedagógica, por tanto, hunde sus raíces en las esencias mismas de la educación emocional e inclusiva, convirtiéndose en punto de inflexión preferente con el que proporcionar atención educativa al colectivo de estudiantes con discapacidad, manteniéndose esta disyuntiva en la Educación Superior.

Teniendo presente la heterogeneidad que define el espacio áulico universitario, la psicopedagogía constituye un motor propulsor de un cambio educativo con miras a enfatizar el valor de la alteridad como elemento definitorio de cada uno de nosotros, siendo determinante responder a situaciones de discriminación con las que puedan encontrarse los estudiantes con diversidad. No cabe olvidar, bajo esta consideración, que se trata de un colectivo que, de definirse como alumnado con necesidades específicas de apoyo educativo durante su escolaridad obligatoria, pasa a considerarse en persona en riesgo de exclusión social y laboral al entrar en la edad adulta, lo que hace que la trayectoria formativa en la universidad sea un pilar esencial para superar barreras en el acceso al mercado laboral.

Trabajar la resiliencia, el empoderamiento y la conformación de una identidad personal consistente y equilibrada se convierten, así pues, en ingredientes esenciales en la intervención emprendida por los profesionales de la psicopedagogía en el escenario universitario, a lo que podría sumarse todo un conjunto de actuaciones fundamentales orientadas a propiciar una plena inclusión social.

Todo ello hace de esta disciplina un instrumento propulsor de la autodeterminación de las personas con diversidad funcional, que implica, además de lograr la capacidad de elegir por uno mismo, descubrir una nueva forma de vivir, y, sobre todo, de alcanzar una verdadera felicidad (Vega, Álvarez-Aguado, Spencer y González-Carrasco, 2020).

Tradicionalmente, el modelo de enseñanza-aprendizaje adoptado con el alumnado catalogado dentro del grupo de personas con necesidades especiales presentaba una estructura rígida, en la que la participación activa del estudiantado quedaba supeditada, en la mayor parte de los casos, a la voluntad y decisiones adoptadas por el profesorado. Sin embargo, el fomento de la autodeterminación en los espacios educativos, implica un trasvase de responsabilidades y compromisos a asumir en las personas con diversidad funcional, llegando a tomar las riendas de su propia vida, y, por ende, de su propio proceso de aprendizaje y formación, lo que, pese a investigarse con mayor frecuencia en el colectivo adolescente (López-Cassá, Pérez-Escoda y Alegre, 2018), resulta trascendente tomarlo en consideración en el caso de los estudiantes universitarios (Chen, 2015; Tomás y Gutiérrez, 2019).

En definitiva, resulta necesario que el profesional de la psicopedagogía adopte una mirada inclusiva y proponga planes de atención a la diversidad lo más acordes con las necesidades 
que presente el alumnado al que atender, incluido, como no podría ser de otro modo, el universitario. La labor del psicopedagogo, por ello, se torna inseparable de la inclusión, buscando esclarecer y superar fórmulas discriminatorias para, en su lugar, conceder plena voz a los discentes, convertidos en verdaderos artífices de su propia vida y su propia historia.

\section{LA INSERCIÓN LABORAL DE PERSONAS CON DISTINTAS CAPACIDADES EN ENTORNOS CULTURALES DESDE LA EDUCACIÓN SUPERIOR}

Entre algunos de los principales desafíos de la universidad del siglo XXI, figura la necesidad de ofrecer una formación comprometida con la diversidad (Melero, Moriña y Perera, 2019; Moriña, 2019, 2020; Rodríguez-Herrero, Izuzquiza y Cabrera, 2020; Tomé, 2017), que lleve a la conformación de estructuras curriculares flexibles en las que, a partir de metodologías innovadoras, los estudiantes con dificultades tengan la posibilidad de acceder al conocimiento en un contexto de aprendizaje compartido, desembocando, todo ello, en una positiva inclusión social y laboral.

Aunque la igualdad de oportunidades constituye un derecho aceptado prácticamente por todos los países, la inserción laboral de las personas con diversidad funcional constituye un reto, que se acrecienta cuando nos referimos a la diversidad funcional intelectual (Munuera, 2016; Riaño-Galán, García-Ruiz, Rodríguez-Martín y Álvarez-Arregui, 2016), al tener que hacer frente a un mayor número de barreras estructurales y actitudinales, que lleva a considerar a este colectivo con menos posibilidades de enfrentarse con éxito a las demandas del mercado laboral.

En contraposición a esta tendencia, la filosofía de la educación inclusiva aboga por la necesidad de subrayar las capacidades en lugar de estigmatizar las dificultades, lo que ha llevado a la emergencia de cursos formativos destinados a "entrenar" a jóvenes adultos con diversidad funcional intelectual para la vida adulta en todos sus ámbitos.

$\mathrm{Al}$ amparo de estas pretensiones, en los niveles educativos no universitarios, la Ley Orgánica de Educación en el año 2006 crearía los Programas de Cualificación Profesional Inicial, aunque no sería hasta el año 2013 cuando la Ley Orgánica de Mejora de la Calidad Educativa, propondría los Ciclos de Formación Profesional Inicial, denominación que sigue vigente en la actualidad (Negri y Leiva, 2016).

Estos programas tratan de ofrecer al alumnado una formación práctica, en la que el trabajo autónomo y la adquisición de herramientas con las que alcanzar una sólida formación en niveles educativos postobligatorios, resultan claves a lo largo de toda la intervención didáctica. 
No obstante, los resultados de diversas investigaciones permiten entrever que estos ciclos formativos no presentan toda la flexibilidad y eficacia para el alumnado con diversidad funcional intelectual (Jurado, Olmos y Pérez, 2015; Negri y Leiva, 2016, 2019), siendo indispensable la reformulación de prácticas docentes, con las que despertar la motivación e interés de este alumnado y permanecer en actualización constante con las corrientes del marco laboral.

Teniendo presentes, por tanto, las carencias de las propuestas formativas no universitarias para el colectivo de personas con diversidad funcional intelectual en su inclusión laboral, la Educación Superior ha tratado de impulsar iniciativas que puedan paliar este tipo de situaciones. Claro reflejo de todo ello es el Título Propio de Técnico Auxiliar en Entornos Culturales implantado en la Universidad de Málaga, cuyas señas de identidad se resumen en la aceptación, comprensión y apoyo a los estudiantes en términos de accesibilidad, adaptación y facilitación de recursos, en un espacio universal de aprendizaje en el que se trata de brindar una atención educativa en consonancia con las características y situaciones personales.

Esta oferta formativa integra competencias transversales determinantes para la vida, el acceso al mundo laboral, y también para la conformación de la identidad personal, lo que lleva a definirla como un medio de inclusión y convivencia, en el que el aprendizaje se funde con la socialización y el crecimiento (Martínez-Silvente y Rodríguez-Infante, 2019).

Teniendo presentes los principios educativos en los que se fundamenta el Título al que venimos haciendo referencia, es importante poner de relieve que, en la actualidad, uno de los espacios de intervención más interesantes desde los que fomentar una educación en valores y potenciar las oportunidades laborales de las personas con diversidad funcional intelectual, queda representado en el mundo del arte.

Este constituye un pretexto pedagógico al servicio del empoderamiento de las personas con diversidad funcional intelectual, que, además de incrementar la motivación e interés, hace despertar lo sensitivo, ayudando a expresar emociones, construir una imagen positiva de uno mismo, trabajar en equipo y desarrollar competencias esenciales con las que desenvolverse en la sociedad de la información y en el escenario laboral (Comeras, Raposo y de Luxan, 2017; Riaño-Galán, García-Ruiz, Rodríguez-Martín y Álvarez-Arregui, 2016).

Temáticas como la poesía, la pintura o la escultura, no solo persiguen aportar los conocimientos necesarios a estos futuros profesionales de entornos culturales, sino además satisfacer, a través de sus técnicas, las necesidades propias de las personas con este tipo de diversidad.

En la línea de lo anterior, por tanto, resulta relevante el Título Propio comentado desde el inicio de este trabajo, cuyo objetivo no es otro que fomentar la inserción laboral de este 
colectivo en Málaga, como símbolo de los avances que aún están por llegar en muchos otros contextos universitarios en busca de la inclusión laboral de las personas con diversidad funcional intelectual en el mundo artístico, y en el que las aportaciones de la psicopedagogía resultan cruciales.

\section{APLICACIÓN PRÁCTICA DE LA PSICOPEDAGOGÍA EN LA ATEN- CIÓN A LA DIVERSIDAD EN EL CONTEXTO UNIVERSITARIO}

El desarrollo de una intervención psicopedagógica se relaciona estrechamente con conceptos tan esenciales como el análisis, la planificación, el seguimiento y la modificación de procesos educativos, lo que lleva a exponer en las páginas siguientes algunos de los pasos más significativos por los que hay que transitar en lo que a las aportaciones de la psicopedagogía se refiere en el plano de la atención a la diversidad en el contexto universitario.

\subsection{Descripción y análisis del contexto de intervención}

La planificación y ejecución de una intervención psicopedagógica constituye un reto, que se acrecienta al dirigirse al contexto universitario, dada la marcada heterogeneidad de alumnado que forma parte del mismo. Todo este proceso, queda supeditado a la dedicación de los profesionales encargados de diseñar la intervención, quienes se esfuerzan en buscar nexos de unión entre la propuesta y aspectos tan importantes como las características de los destinatarios y sus principales necesidades.

En la actualidad, trasladar los fundamentos de la psicopedagogía a las aulas universitarias va acompañado de la necesidad de asumir una mirada inclusiva, solidaria y respetuosa con la diversidad (Baraldi, 2016), siendo indispensable llevar a cabo un estudio detenido del contexto en el que implementar la intervención, para delimitar las carencias, establecer prioridades y formular objetivos realistas.

En este trabajo, la contextualización es especialmente necesaria, dado que se hace referencia a una intervención psicopedagógica diseñada durante el periodo de prácticas de una estudiante del Máster en Psicopedagogía de la Universidad de Málaga, que, como parte de su proceso formativo, se encuentra inmersa en la elaboración de un proyecto teórico-práctico.

Dicho proyecto se desarrolla en un espacio de aprendizaje universitario, cuyos protagonistas son estudiantes mayores de edad, a los que se trata de ofrecer experiencias educativas con un alto componente socio-laboral. En concreto, se trata de trece estudiantes, diez mujeres 
y tres hombres, con diversidad funcional intelectual cursando el Título Propio de Técnico Auxiliar en Entornos Culturales impartido en la Facultad de Ciencias de la Educación de la Universidad de Málaga (España) durante el curso académico 2019-2020.

Esta titulación, de carácter no oficial, se enmarca dentro de los programas universitarios de formación para el empleo de jóvenes con diversidad funcional intelectual, en el marco de la convocatoria de ayudas de Fundación ONCE, contando con el apoyo financiero del Fondo Social Europeo, a través del Programa Operativo de Empleo Juvenil.

Consta de 45 créditos equivalentes a 450 horas de trabajo, de los que 40 se destinan a docencia teórico-práctica, mientras que el resto comprenden prácticas externas en centros culturales y museos de Málaga, como el Ateneo, el Contenedor Cultural de la Universidad de Málaga, el Centro de Arte Contemporáneo, el Museo Picasso, y el Vicerrectorado de Estudiantes, representando, todos ellos, una excelente oportunidad para abrir las puertas de la universidad a la realidad cultural, buscando la excelencia hacia la inclusión educativa y laboral de personas con diversidad funcional intelectual, siendo el arte el hilo conductor que vertebra su tejido curricular.

En la tabla 1, se aportan de forma gráfica los diferentes módulos de aprendizaje que lo constituyen.

Tabla 1. Módulos de aprendizaje del título de Técnico Auxiliar en Entornos Culturales de la Universidad de Málaga

MÓDULO 1. DESARROLLO EMOCIONAL Y SOCIAL

Taller de Habilidades sociales.

Desarrollo de Habilidades Emocionales

Estrategias de aprendizaje.

MÓDULO 2. COMUNICACIÓN ORAL Y ESCRITA

Lectoescritura.

Taller de comunicación oral.

MÓDULO 3. ORIENTACIÓN LABORAL

Orientación Laboral. Inserta Empleo

MÓDULO 4. HERRAMIENTAS EN ENTORNOS CULTURALES

Los recursos tecnológicos en los entornos culturales.

Herramientas digitales: softwares, webs, redes sociales.

MÓDULO 5. PATRIMONIO CULTURAL

Museología y Museografía.

Patrimonio cultural y artístico. 


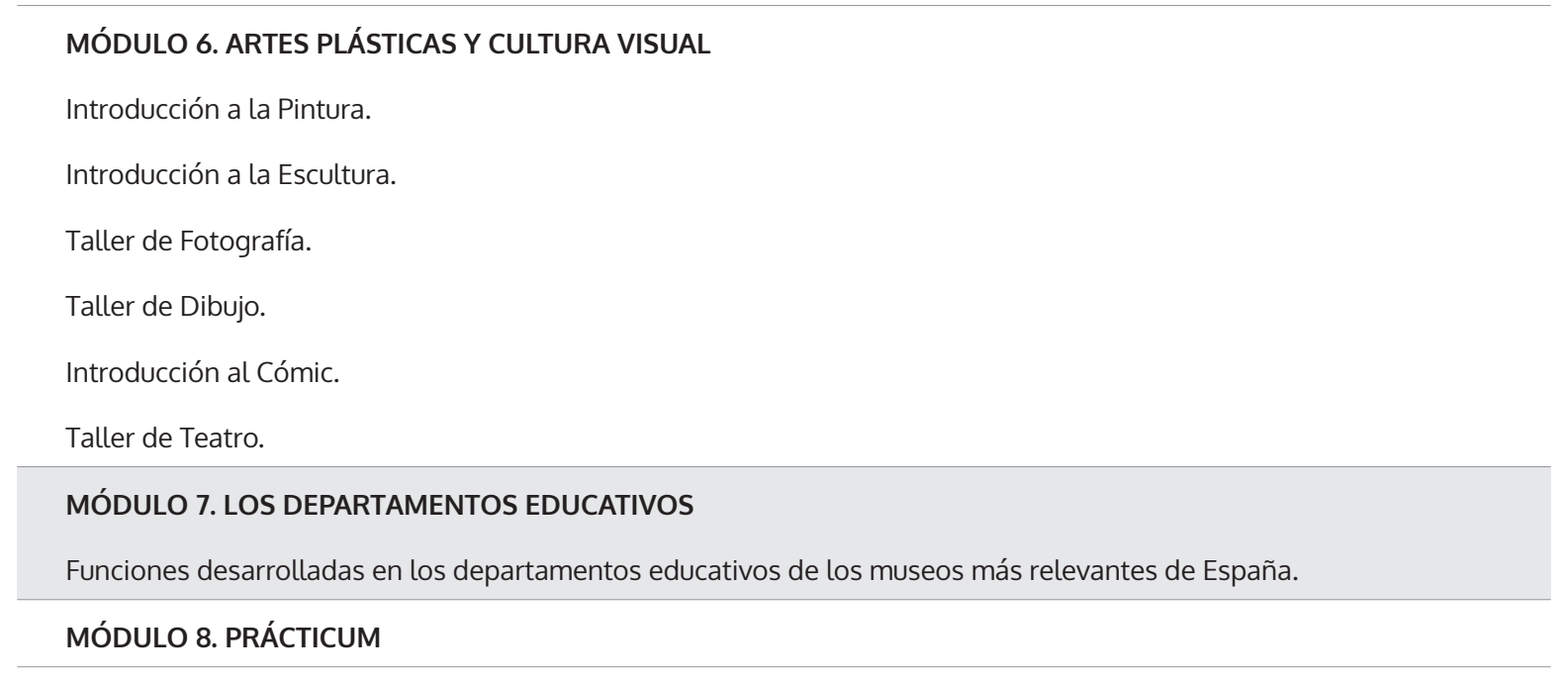

Fuente: Elaboración propia a partir de Martínez- Silvente y Rodríguez-Infante, 2019

El abordaje de los módulos teóricos se lleva a cabo a partir del desarrollo de ponencias protagonizadas por expertos en las diversas temáticas, buscando cubrir de forma holística los ámbitos de aprendizaje y asumir una concepción constructivista.

No todo el alumnado puede acceder a este Título, dado que es necesario cumplir con ciertos requisitos, entre los que figuran presentar una diversidad funcional intelectual con un grado igual o superior al 33\% reconocida oficialmente, tener una edad comprendida entre los 18 y los 30 años y contar con la inscripción en el Sistema Nacional de Garantía Juvenil previamente al inicio del curso (Martínez-Silvente y Rodríguez-Infante, 2019).

Tan solo trece estudiantes reunían estas características durante el curso académico 20192020, dando como resultado un grupo altamente diverso marcado por la existencia de una amplia variedad de dificultades de índole cognitiva, entre las que figuraba el Trastorno del Espectro Autista, el Trastorno por Déficit de Atención con Hiperactividad y el Síndrome de Down, junto con otras comorbilidades con diversidad funcional sensorial o física.

En la descripción de las características más significativas del grupo hacia el que se dirige la propuesta de intervención psicopedagógica que sustenta este trabajo, es importante poner de relieve la actitud favorable al aprendizaje que define el mismo, marcada por la constancia, el interés, el entusiasmo y el esfuerzo, como componentes esenciales al servicio de la conformación de personas de pleno derecho.

Todo ello ha podido apreciarse gracias al periodo de estudio y elaboración del diseño de intervención psicopedagógica, que estuvo precedido de sesiones dedicadas a la toma de con- 
tacto con el alumnado, recurriendo a la observación sistemática, como técnica cualitativa con la que acceder a las principales características, necesidades y demandas manifestadas por todos y cada uno de los estudiantes, constituyendo, todo ello, verdadero referente de la propuesta.

En ese proceso de identificación de necesidades, pudo advertirse una amplia variedad de casuísticas que no hacen sino reflejar la marcada heterogeneidad del grupo de estudiantes.

Su deseo por aprender se encontraba más arraigado a la acción y la reflexión que a la teoría, es decir, precisaban desarrollar aprendizajes relevantes y significativos, acordes con la realidad y, sobre todo, de aplicación práctica. A ello se unía su inquietud constante por temáticas vinculadas con la igualdad de género, lo que los llevaba al deseo constante de indagar sobre la existencia de artistas femeninas más allá de las reconocidas mundialmente.

El mundo de las letras, y, en especial, la poesía, parecía ser del agrado de estos estudiantes, si bien pudo denotarse que no se le había prestado la atención merecida, lo que se vislumbró como una gran oportunidad de aprendizaje.

De la misma forma, el alumnado presentaba inquietud relacionada con los períodos de prácticas, interrogando de forma recurrente los compromisos y tareas a asumir, con cierta dosis de incertidumbre.

Finalmente, se pudo constatar que el grupo presentaba grandes limitaciones para trabajar de forma cooperativa, lo que se percibió como aspecto a reforzar desde una vertiente psicopedagógica.

Dadas las situaciones anteriormente descritas, fue diseñada una propuesta de intervención en la que, además de tener presentes los principios de aceptación, comprensión y apoyo, se sentaron las bases de una temática focalizada en las llamadas "Sinsombrero", integrada por artistas femeninas pertenecientes a la Generación del 27, que constituyeron una fructífera oportunidad en la trayectoria formativa de este grupo de estudiantes.

\subsection{La psicopedagogía como herramienta al servicio del empoderamiento de las perso- nas con diversidad funcional intelectual en la Educación Superior}

El actual modelo universitario ante el que nos encontramos, se define, entre otros aspectos, por la calidad y la acreditación, contemplando nuevos escenarios formativos con los que responder a las necesidades sociales y los emergentes escenarios profesionales, trabajando en clave inte- 
gral y humanística (Pérez y Castaño, 2016), para formar futuros ciudadanos capaces de convivir, desarrollar un espíritu crítico y con una actitud favorable a la formación a lo largo de la vida.

Aunque en las últimas décadas han sido muchos los estudios realizados para valorar el impacto del llamado Espacio Europeo de Educación Superior, han sido escasas las investigaciones en las que se analiza la importancia de esta etapa educativa en el empoderamiento de personas con diversidad funcional intelectual (Izuzquiza y Rodríguez-Herrero, 2016). Precisamente desde este ámbito de actuación la psicopedagogía cobra un papel esencial, puesto que, no solo proporciona el apoyo necesario para solventar necesidades, sino que, además, trata de prever posibles dificultades en la adquisición de aprendizajes.

En esta línea de pensamiento se sitúa la intervención psicopedagógica de este trabajo, titulada "Derrumbando las barreras de la desigualdad a través del arte", orientada a la profesionalización de personas con diversidad funcional intelectual para su inclusión en el ámbito socio-laboral de la cultura.

La emergencia de esta intervención y su diseño parte de todas aquellas necesidades e intereses, anteriormente descritos, y detectados en el grupo-clase que constituyó el Título Propio de Técnico Auxiliar en Entornos Culturales de la Universidad de Málaga durante el curso académico 2019-2020. Su planificación, por tanto, obedece a estas características contextuales, además de a los aspectos formales exigidos durante el proceso de creación de dicha intervención, dictados por el propio Máster en Psicopedagogía de la universidad malagueña.

La propuesta consta de una organización espacio-temporal concisa, cuyas dinámicas conjugan la teoría con la práctica, en orden a propiciar la construcción de aprendizajes significativos. Su principal sustento es hacer realidad una educación inclusiva, en la que estén presentes los principios de equidad y respeto, para responder de la mejor forma posible a las necesidades del alumnado al que va destinada, sin subestimar sus capacidades.

Para cumplir con este propósito y, tomando como referencia la titulación mencionada y su temática, la decisión de tomar como enlace el mundo del arte puede justificarse teniendo en cuenta que constituyó un pretexto para responder a los intereses del alumnado destinatario, de la misma forma que se tuvo en cuenta su gran potencial pedagógico para trabajar la inclusión social, el empoderamiento y la resiliencia de las personas con diversidad funcional (De la Chica, 2017).

En concreto, la propuesta de intervención psicopedagógica tuvo como principal hilo conductor un movimiento artístico de gran arraigo como fue el integrado por mujeres artistas 
representantes de la Generación del 27 en España, bajo el apelativo "Sinsombrero". Entre algunas de sus figuras más representativas cabe citar a María Blanchard o Maruja Mallo, quienes, entre los años 1898 y 1914, al contrario que sus análogos masculinos, vieron truncados sus éxitos artísticos por el mero hecho de pertenecer al género “equivocado” (Balló, 2016).

Su denominación conforma un modo de percibir a estas mujeres como aquellas que se atrevieron a retar, en cierta medida, a la sociedad y a la época histórica que les tocó vivir, desprendiéndose de una prenda que formaba parte de la identidad femenina del momento: el sombrero, en un intento por reivindicar su rol y dejar de permanecer en el olvido.

Siguiendo las bases psicopedagógicas de la orientación educativa y, dado el origen de la propuesta, es importante poner de relieve que la intervención que se presenta se enmarca en un modelo de servicios llevado a cabo mediante un programa (Bausela, 2004).

Esto supone que, con carácter previo a la planificación, se realizó una detección de necesidades y un estudio en profundidad de las características más significativas del grupo receptor, siendo el diseño resultante aquel con el que satisfacer de forma holística a las personas beneficiarias, desde sus voces, su mirada y su implicación continua (Tallón et al., 2019).

En la tabla 2 de recogen los objetivos que han formado parte de esta propuesta y los conceptos correspondientes.

Tabla 2. Relación de objetivos y conceptos programados en la intervención

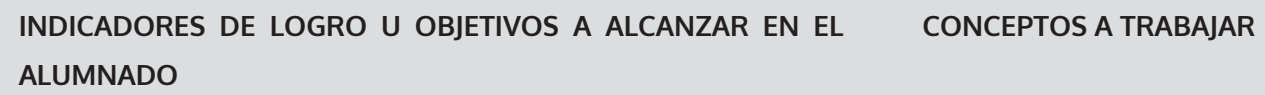

Ampliar el conocimiento sobre el movimiento artístico de la Generación del 27, con especial incidencia en las "Sinsombrero".
La Generación del 27 desde sus protagonistas femeninas, las llamadas "Sinsombrero".
Demostrar actitudes y habilidades de autodeterminación, identificando virtudes y defectos desde el plano de un Técnico Auxiliar de Entornos Culturales.

Aceptar la autonomía y responsabilidad en la toma de decisiones, adoptando un comportamiento flexible ante las situaciones que puedan surgir durante la trayectoria formativa o futuro escenario laboral.
El museo y su funcionamiento: Actividades a desarrollar como técnicos/as auxiliares en entornos culturales. 
Adquirir y expresar habilidades socioemocionales mediante experiencias de aprendizaje vivencial, significativo y cooperativo.

Desarrollar habilidades artísticas, no solo limitadas al mundo de la Educación Plástica y Artística, sino también en torno a la escritura.
La inclusión: Percibir la diversidad más allá de la discapacidad.

Desarrollar la comprensión y empatía hacia las necesidades que puedan presentar otras personas con diversidad.

La profesionalidad: Promover diferentes situaciones y escenarios que, desde la perspectiva

Reconstruir las ideas propias, generando un pensamiento crítico fundamentado, que permita generar un posicionamiento concreto de un técnico auxiliar, impliquen la asunción de sobre temas de cariz social, cultural y artístico.

Fuente: Elaboración propia

Para favorecer la consecución de cada uno de los objetivos y el tratamiento didáctico de los conceptos, se ha tenido en cuenta la disponibilidad horaria de la titulación, que comprende cuatro sesiones de clase semanales, estableciendo una temporalización que se extiende a lo largo de dos meses, trabajando en cada uno de ellos, cuatro sesiones.

En la tabla 3 se ofrece una delimitación de las sesiones que constituyeron la intervención.

Tabla 3. Sesiones de la intervención psicopedagógica diseñada

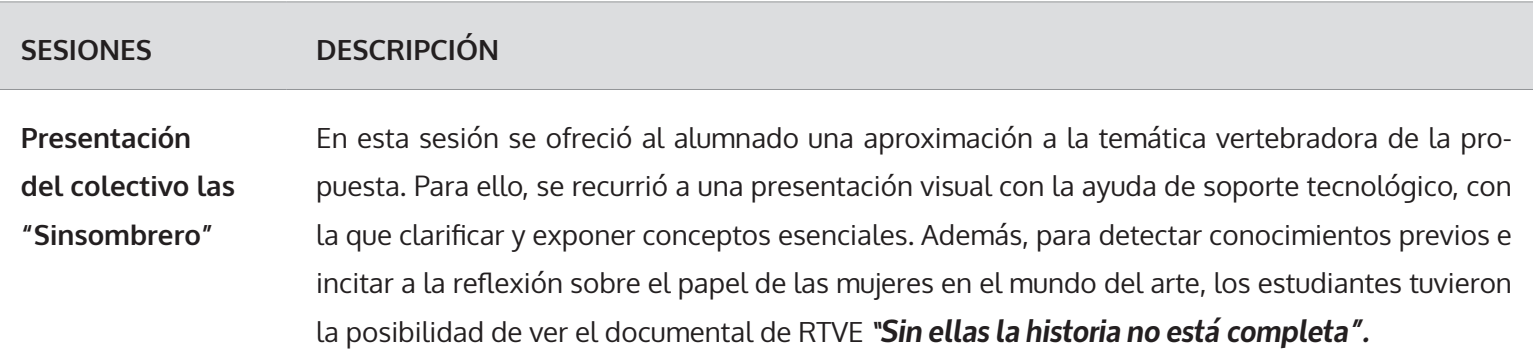

María Zambrano, Durante esta sesión, se trabajó en torno a la figura de María Zambrano, partiendo de una presenla poetisa mala- tación gráfica con alto componente visual. Posteriormente, se trató de dar voz a su obra mediante gueña un recital de poesía por parte del alumnado, para, finalmente, introducir al mismo en la creación autónoma de su propia composición, gracias a una guía de pensamiento ilustrada en la figura 1. 
María Blanchard, la pintora cubista
A lo largo de esta sesión, se trabajó acerca de la vida y obra de María Blanchard, recurriendo a las herramientas digitales como forma de establecer diferencias entre sus obras cubistas y otras pertenecientes a Pablo Picasso.

Para propiciar experiencias de aprendizaje compartido, se optó por la realización de pequeños proyectos de investigación en los que, grupos de tres componentes, siguiendo la técnica del Puzle de Aronson, se encargaron de investigar una obra de esta artista para su posterior exposición al grupo-clase.
Al objeto de trabajar en torno a las composiciones escultóricas, se optó por analizar la obra de Margarita Gil Roësset, a partir de una presentación interactiva inserta en la plataforma Genially. Seguidamente, se prosiguió con la creación de una historia conjunta partiendo de la inspiración de su escultura, para finalizar con el diseño personal de obras escultóricas con arcilla.
Maruja Mallo, el retrato de una rebelde
Para conocer a fondo la obra de esta artista, los estudiantes tuvieron la oportunidad de trabajar la técnica del retrato, a partir de la creación de un autorretrato sobre pintura acrílica. La finalidad transversal de esta actividad iba destinada a potenciar la autoestima, el autoconcepto y la autodeterminación, a partir del diseño personal de la imagen de uno mismo.
El arte en todas sus formas
En esta ocasión, se seleccionaron obras de las artistas más aclamadas por los propios estudiantes, pertenecientes al género literario, escultórico o pictórico, para que, de forma individual, se asignara a la obra en cuestión una canción, una imagen e incluso una poesía, relacionando el arte en todas sus magnitudes.

Con posterioridad, cada alumno se encargó de su exposición al grupo-clase, a partir de presentaciones sencillas en formato audiovisual, lo que permitió trabajar de forma transversal las competencias digitales.
Preparación de la presentación final

Con la finalidad de impartir una última conferencia con la que dar a conocer el movimiento artístico de las "Sinsombrero" por parte del propio alumnado, se propuso una sesión dedicada a organizar la misma. El objetivo fue constatar los aprendizajes alcanzados durante el transcurso de las diferentes sesiones de trabajo y ofrecer recursos para planificar y organizar la conferencia, además de autoinstrucciones, necesarias para desenvolverse de forma eficaz ante nuevas e inesperadas situaciones, como se ilustra en la figura 2.

Esta sesión se dedicaría a impartir la conferencia anteriormente comentada, dirigida tanto a las

Conferencia

"Descubriendo a

Las Sinsombrero,

la generación

olvidada" familias del alumnado, como especialmente, al alumnado universitario, obteniendo la oportunidad de conocer este movimiento artístico gracias a sus compañeros/as con diversidad.

Fuente: Elaboración propia 
Figura 1. Guía de pensamiento para la escritura de poesía. Fuente: Elaboración propia

- Nos sentamos en una posición cómoda y creamos un
ambiente relajante y placentero.
- Cogemos el lápiz y en un papel en sucio vamos escribiendo todo aquello
que nos vaya viniendo a la cabeza.
- De todo lo escrito escogemos aquellas palabras que suenen bien juntas
y rimen. Por ejemplo: risa y camisa.
- Escribimos varias frases para así iniciar la poesía, buscando
el ritmo adecuado.
- Las releemos en voz alta.
- Corregimos aquello que no nos guste.
- Se la enseñamos a nuestro/a amigo/a, familia, etc.
- Tras ello, la repasamos y cambiamos lo que no nos guste
o nos recomienden. iY listo!

Figura 2. Autoinstrucciones para propiciar la adaptación a nuevas situaciones. Fuente: Elaboración propia

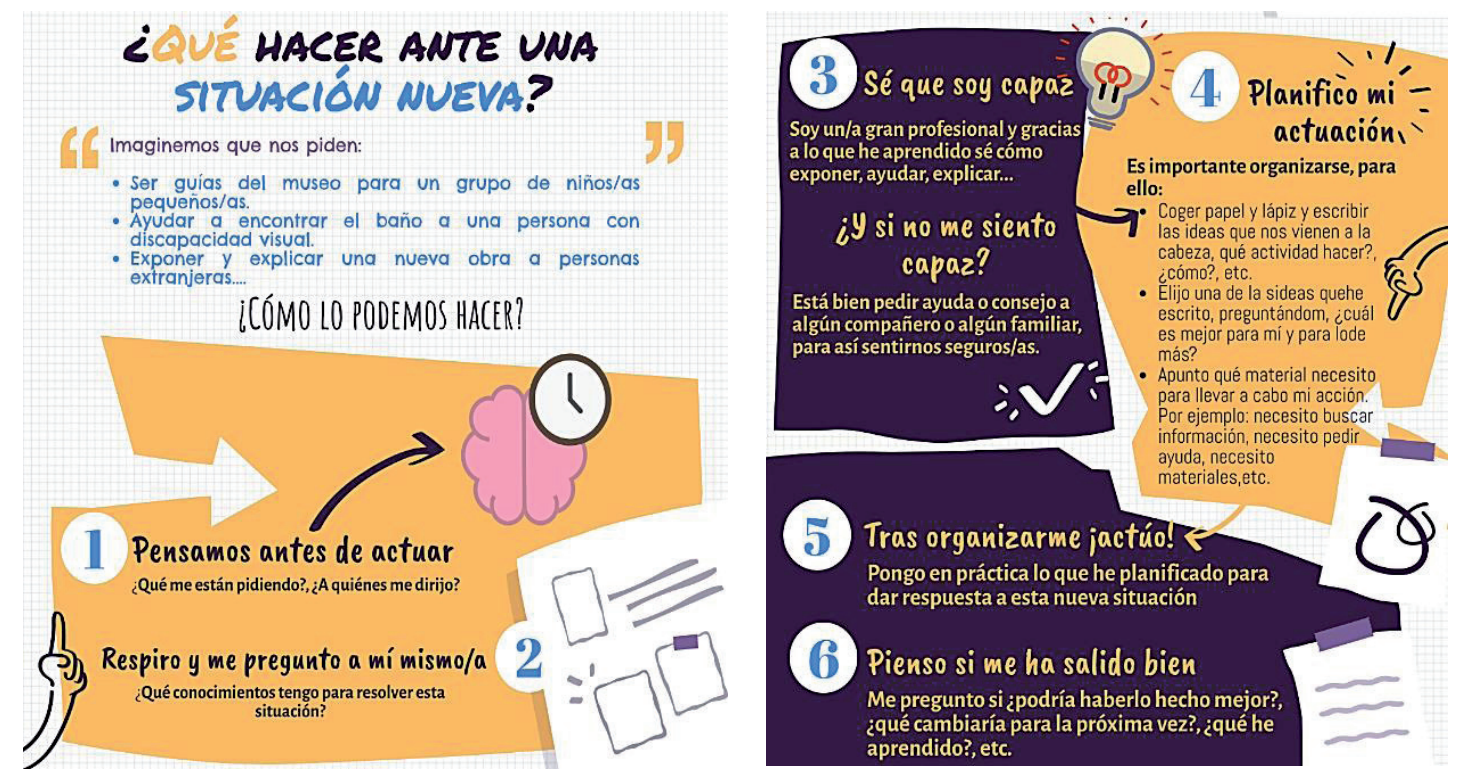

Como puede apreciarse, a lo largo de todas las sesiones se han tenido en cuenta las características del alumnado, sus intereses y demandas, procurando que existiera un cierto equilibrio entre los diversos aspectos abordados. Al tratarse de un colectivo con dificultades para mantener la atención durante espacios prolongados de tiempo, se han incluido actividades dinámicas que reclamaban una participación e implicación activa, recurriendo, en la mayor parte de los casos, a apoyos visuales, estimados necesarios para trabajar aspectos referentes a las manifestaciones artísticas. 
En términos de convivencia, las sesiones de trabajo han propiciado la conformación de momentos de encuentro y han hecho del espacio áulico un lugar vivo de aprendizaje, concediendo plena voz al alumnado destinatario, asumiendo, de forma explícita, que la educación universitaria debe brindar a las personas con diversidad la oportunidad de incluirse en la experiencia sociocultural que implica la acreditación como estudiante universitario.

De la misma forma, a lo largo de toda la intervención se ha recurrido a estrategias que pueden resultar esenciales e imprescindibles para garantizar el empoderamiento del colectivo de estudiantes con diversidad funcional intelectual a lo largo de su vida laboral en entornos culturales, buscando, de forma fehaciente, proporcionar las herramientas necesarias con las que desenvolverse de manera autónoma a lo largo de su vida profesional.

\section{CONCLUSIONES}

Resulta imposible describir el impacto personal y social que ha conllevado la puesta en práctica de esta intervención psicopedagógica. No solo por su facilidad para dar voz a un movimiento artístico en pro de la igualdad de género como el de "Las Sinsombrero", sino también por el protagonismo y la autodeterminación que han podido alcanzar los estudiantes con diversidad funcional intelectual que han formado parte de esta experiencia.

Son vivencias de este tipo las que permiten reivindicar que la vida de las personas con diversidad prosigue más allá de la educación obligatoria, por lo que tienen el derecho de recibir la formación que les otorgue las herramientas para una plena inclusión socio-laboral.

$\mathrm{El}$ arte, de la misma forma, ha sido un recurso psicopedagógico determinante con el que percibir en la diversidad un valor incalculable de la realidad formativa universitaria, con el que la inclusión educativa se proyecta de forma decidida y responsable.

$\mathrm{Y}$ es que, en el caso concreto de las personas con diversidad funcional intelectual, la cultura puede jugar un papel imprescindible en su conducta, puesto que las manifestaciones artísticas conllevan implicaciones emocionales y por supuesto, un componente social y culturalmente construido que requiere de un mayor trabajo por parte de las mismas para poder llegar a ser comprendido.

El ámbito artístico, en definitiva, se nos muestra cada vez más recíproco a la hora de aceptar entre sus representantes a personas con capacidades diversas, que luchan por reclamar los derechos de toda una comunidad, históricamente olvidada y rechazada por la sociedad. 
En este escenario, la psicopedagogía se esfuerza de forma fehaciente en hacer realidad la inclusión educativa, social y laboral, al tiempo que refleja la evolución y el compromiso asumido por los centros universitarios en la creación de espacios de aprendizaje enriquecedores, sostenibles e inclusivos.

\section{REFERENCIAS BIBLIOGRÁFICAS}

Balló, T. (2016). Las Simsombrero. Barcelona: Espasa Libros.

Baraldi, C. (2016). Psicopedagogía hoy. Revista Pilquen, 13(1), 51-61.

Bausela, E. (2004). Modelos de orientación e intervención psicopedagógica; modelo de intervención por programas. EduPsykhé, 3(2), 201-216

Bortolini, R., Oscar, F. y Almeida, I. (2018). Inclusão no ensino superior: a percepção de docentes de una instituição pública do interior do estado de São Paulo. Psicología Escolar e Educacional, SP, 127-134. http://dx.doi.org/10.1590/2175-3539/2018/010

Carballo, R., Cotán, A. y Spínola, Y. (2019). An Inclusive Pedagogy in Arts and Humanities University Classrooms: What faculty members do. Art and Humanities in Higher Education, 1-21. https:// doi.org/10.1177/1474022219884281

Cejudo, J. (2017). Competencias profesionales y competencias emocionales en orientadores escolares. Profesorado. Revista de Currículum y Formación de Profesorado, 21(3), 349-370.

Chen, C. (2015). Incremental validity of achievement goals in predicting subjective well- being among university students. Fournal of Cognitive Education and Psychology, 14(1), 38-62. https://dx.doi. org/10.1891/1945-8959.14.1.38

Collins, A., Azmat, F. y Rentschler, R. (2018). Bringing Everyone on the Same Journey: Revisiting Inclusion in Higher Education. Studies in Higher Education, 1-13. https://dx.doi.org/10.1080/030 75079.2018.1450852.

Comeras, A.B., Raposo, J.F. y de Luxan, M. (2017). Expresiones artísticas de personas con Discapacidad Intelectual y su relación con estrategias cognitivas arquitectónicas. Ediciones Complutense, 29, 175-189. https://dx.doi.org/10.5209/ARIS.53960

De la chica, L. (2017). Nuevas metodologías pedagógicas: Arte y discapacidad. Revista Internacional de Apoyo a la Inclusión, Logopedia, Sociedad y Multiculturalidad, 3(1), 183-197. 
Demchenko, I., Rokosovyk, N. y Volodymyr, S. (2017). Psycho-pedagogical diagnostics of inclusive competence of future professionals in Pedagogical field. Science and Education, 8, 141-149. https://dx.doi.org/10.24195/2414-4665-2017-8-21

Fernández-Fernández, I.M., Véliz-Briones, V. y Ruiz-Cedeño, A.I. (2016). Hacia una cultura pedagógica inclusiva. Experiencias desde la práctica universitaria. Revista Electrónica Educare, 20(3), 1-15. http://dx.doi.org/10.15359/ree.20-3.13

García, M. G. y García, M. J. C. (2017). La contribución de la universidad al desarrollo de prácticas inclusivas: dilemas y propuestas para avanzar compartiendo. Revista de Educación Inclusiva, 5(1), 123-138.

Izuzquiza, D. y Rodríguez-Herrero, P. (2016). Inclusion of people with intellectual disabilities in university. Results of the Promentor Program (UAM-PRODIS, SPAIN). Siglo Cero, 47(4), 27-43. http://dx.doi.org/10.14201/scero20164742743

Jurado, P., Olmos, P. y Pérez, A. (2015). Los jóvenes en situación de vulnerabilidad y los programas formativos de transición al mundo del trabajo. Educar, 51(1), 211-224. http://dx.doi.org/10.5565/ rev/educar.648

Lawrie, G., E. Marquis, E. Fuller, T. Newman, M. Qiu, M. Nomikoudis, F. Roelofs, L. y van Dam, H. (2017). Moving Towards Inclusive Learning and Teaching: A Synthesis of Recent Literature. Teaching \& Learning Inquiry, 5(1): 1-13. https://dx.doi.org/10.20343/teachlearninqu.5.1.3

López-Cassá, E., Pérez-Escoda, N. y Alegre, A. (2018). Competencia emocional, satisfacción en contextos específicos y satisfacción con la vida en la adolescencia. Revista de Investigación Educativa, 36(1), 57-73. https://dx.doi.org/10.6018/rie.36.1.273131

Lorente, E. y Sales, A. (2017). Perfil y funciones del psicopedagogo en el contexto de una escuela inclusiva. Revista Nacional e Internacional de Educación Inclusiva, 10(1), 117-132.

Martínez- Silvente, M.J. y Rodríguez- Infante, G. (2019). Formación accesible en el ámbito de la cultura y el patrimonio de las personas con discapacidad intelectual: el caso de la Universidad de Málaga. Repositorio institucional de la Universidad de Málaga

Melero, N. Moriña, A. y Perera, V.H. (2019). Acciones del profesorado para una práctica inclusiva en la universidad. Revista Brasileira de Educação, 24, 1-19. https://dx.doi.org/10.1590/S1413$\underline{24782019240016}$

Messi, L., Rossi, B. y Ventura, A.C. (2016). La Psicopedagogía en el ámbito escolar: ¿Qué y cómo representan los docentes la intervención psicopedagógica? Perspectiva Educacional. Formación de Profesores, 55(2),110-128. https://dx.doi.org/10.4151/07189729-Vol.55-Iss.2.Art.409 
Moriña, A. (2019). The keys to learning for university students with disabilities: motivation, emotion and faculty-student relationships. PLoS ONE, 14(5), 1-15. https://dx.doi.org/10.1371/journal. pone. 0215249

Moriña, A. (2020). Faculty members who engage in inclusive pedagogy: Methodological and effective strategies for teaching. Teaching and Higher Education, 1-16. https://doi.org/10.1080/13562517 .2020 .1724938

Munuera, M.P. (2016). Inclusión sociolaboral de las personas con discapacidad. Mediación laboral. Mediaciones Sociales, 14,161-179. http://dx.doi.org/10.5209/rev MESO.2015.n14.51564

Negri, M.I y Leiva, J.J. (2016). El papel de la formación profesional en la inclusión socio- laboral de jóvenes con Diversidad Funcional Intelectual. Revista nacional e internacional de Educación Inclusiva, 9(3), 13-28.

Negri, M.I. y Leiva, J.J. (2019). Liderazgo de los docentes de formación profesional básica para la mediación escuela-empleo del alumnado con diversidad funcional intelectual. Contextos Educativos, 24, 95-111. http://dx.doi.org/10.18172/con.3902

Pérez, S. y Castaño, R. (2016). Funciones de la Universidad en el siglo XXI: humanística, básica e integral. Revista Electrónica Interuniversitaria de Formación del Profesorado,19(1),191- 199. http://dx.doi.org/10.6018/reifop.19.1.20245

Riaño-Galán, A., García-Ruiz, R., Rodríguez-Martín, A. y Álvarez-Arregui, E. (2016). Calidad de vida e inserción socio-laboral de jóvenes con discapacidad. REDIE: Revista Electrónica de Investigación Educativa, 18(1), 112-127.

Rodríguez Herrero, P., Izuzquiza, D. y Cabrera, A. (2020): Inclusive education at a Spanish University: the voice of students with intellectual disability Disability \& Society, 1-23. https://dx.doi.org/1 $\underline{0.1080 / 09687599.2020 .1745758}$

Tallón, S., Fernández, C., Hervás, M., Aparicio, M. y Polo, M.T. (2019). La diversidad en la Educación Superior: Programas de intervención actuales. International fournal of Developmental and Educational Psychology, 1(2), 445-454. https://doi.org/10.17060/ijodaep.2019.n1.v3.1517

Tomás, J.M. y Gutiérrez, M. (2019). Aportaciones de la teoría de la autodeterminación a la predicción de la satisfacción académica en estudiantes universitarios. Revista de Investigación Educativa, 37(2), 471-485. http://dx.doi.org/10.6018/rie.37.2.328191

Tomé, M. (2017). Attitudes toward Inclusive Education and Practical Consequences in Final Year Students of Education Degrees. Procedia. Social and Behavioral Sciences, 237, 1184-1188. https:// dx.doi.org/10.1016/j.sbspro.2017.02.187 
Vega, V., Álvarez-Aguado, I., Spencer, H. y González-Carrasco, F. (2020). Avanzando en autodeterminación: Estudio sobre las autopercepciones de personas adultas con discapacidad intelectual desde una perspectiva de investigación inclusiva. Siglo Cero, 51(1), 31-52. https://doi.org/10.14201/ scero20205113152

Ventura, A.C. (2015). Dificultades de aprendizaje y Psicopedagogía: Una perspectiva estilística. Construçao Psicopedagógica, 23(24), 6-19. 